\title{
ON PERIODIC GROUPS HAVING ALMOST REGULAR 2-ELEMENTS*
}

\author{
by N. R. ROCCO and P. SHUMYATSKY
}

(Received 19th August 1996)

\begin{abstract}
We show that if a periodic residually-finite group $G$ has a 2 -element with finite centralizer then $G$ is locally finite.
\end{abstract}

1991 Mathematics subject classification: 20 F50.

\section{Introduction}

An element $a$ of a group $G$ is called almost regular if $C_{G}(a)$ is finite. In some situations existence of an almost regular element in $G$ implies very strong conclusions about $G$. For example Šnkov proved in [14] that a periodic group $G$ having an almost regular element of order two is locally finite (and even contains a solvable subgroup of finite index). Two other proofs of this result can be found in [2] and [7]. Locally finite groups having an almost regular element of arbitrary prime order have been studied intensively in the seventies and eighties (see for example [7]). Khukhro showed that these groups have a nilpotent subgroup of finite index [10]. In general, a very interesting direction in locally finite group theory is to classify in some sense locally finite groups $G$ having a finite subgroup $A$ such that $C_{G}(A)$ possesses some prescribed property, as for example the property to be a linear group [8].

In this paper we are interested in the following question, which is natural in view of the result of Šunkov.

Given a periodic group $G$ with an almost regular element, under what conditions does it follow that $G$ is locally finite?

According to Ol'shanski, for any positive integer $n$ which has at least one odd divisor there exists an infinite group $G$ having an almost regular element of order $n$ such that all proper subgroups of $G$ are finite [12]. Therefore the result of Šunkov cannot be extended to periodic groups having an almost regular element whose order is not a 2-power.

In this paper we consider periodic residually-finite groups having an almost regular element of order $2^{n}$. Note that groups constructed in $[1,4,5,6,13]$ are finitely-generated periodic residually-finite and infinite. We will prove here the following result.

* Supported by CNPq and FAPDF-Brazil 
Theorem A. Let $G$ be a periodic residually-finite group having an almost regular 2-element. Then $G$ is locally finite.

The above theorem will be derived from the following closely related result.

Proposition B. Let $G$ be a periodic residually-nilpotent group admitting a fixedpoint-free automorphism of order $2^{n}$. Then $G$ is locally finite.

\section{Proof of Proposition B}

Let $G$ be a periodic group. For any element $x \in G$ of odd order let us use $x^{\frac{1}{2}}$ to denote the element $y \in\langle x\rangle$ such that $y^{2}=x$. If $G$ is a periodic $2^{\prime}$-group and $\phi$ is an involutory automorphism of $G$ then $x\left(x^{-\phi} x\right)^{-\frac{1}{2}} \in C_{G}(\phi)$. Indeed, let $h=x\left(x^{-\phi} x\right)^{-\frac{1}{2}}$. Note that

$$
\left(x^{-\phi} x\right)^{\frac{1}{2}}=x^{-\phi} x\left(x^{-\phi} x\right)^{-\frac{1}{2}}
$$

We have

$$
h^{\phi}=x^{\phi}\left(x^{-1} x^{\phi}\right)^{-\frac{1}{2}}=x^{\phi}\left(x^{-\phi} x\right)^{\frac{1}{2}}=x^{\phi} x^{-\phi} x\left(x^{-\phi} x\right)^{-\frac{1}{2}}=x\left(x^{-\phi} x\right)^{-\frac{1}{2}}=h
$$

Lemma 2.1. Let $G$ be a periodic group acted on by a finite 2-group A. Suppose that $C_{G}(A)$ contains no 2-element. Then $G$ is a 2'-group.

Proof. We will use induction on $|A|$. Suppose first that $A$ is of order two and let $\phi$ be the involution in $A$. If $G$ contains elements of even order there exists an involution $\tau \in G$. If $\tau \cdot \tau^{\phi}$ is of even order then the involution from $\left\langle\tau \cdot \tau^{\phi}\right\rangle$ is contained in $C_{G}(A)$, a contradiction. Assume that the order of $\tau \cdot \tau^{\phi}$ is odd. Then $\left(\tau^{\phi} \cdot \tau\right)^{\frac{1}{2}} \cdot \tau$ is an involution contained in $C_{G}(A)$.

Let now $|A| \geq 4$ and $\phi$ an involution in $Z(A)$. Set $H=C_{G}(\phi)$. By the preceding paragraph, if $G$ contains elements of even order then so does $H$. Obviously, $H$ is $A$ invariant and $A$ induces a group of automorphisms of $H$ whose order is strictly less than that of $A$. Now the induction hypothesis yields that $C_{H}(A)$ contains elements of even order. The lemma follows.

Lemma 2.2. Let $G$ be a periodic 2'-group acted on by a finite 2-group A. Suppose that $N$ is a normal $A$-invariant subgroup of $G$. Then

$$
C_{G}(A) N / N=C_{G / N}(A)
$$

Proof. Let $|A|=2^{n}$. Using induction on $n$ we will show that 


$$
C_{G}(A) N / N \geq C_{G / N}(A) .
$$

Suppose first that $n=1$ and let $\phi$ be the involution in $A$. Let $x \in G$ and $x N \in C_{G / N}(A)$. Then $x^{-\phi} x \in N$. Since

$$
x=x\left(x^{-\phi} x\right)^{-\frac{1}{2}}\left(x^{-\phi} x\right)^{\frac{1}{2}} \text { and } x\left(x^{-\phi} x\right)^{-\frac{1}{2}} \in C_{G}(\phi),
$$

we obtain that $x N \in C_{G}(A) N / N$.

Let now $n$ be arbitrary and $\phi$ an involution in $Z(A)$. Set $H=C_{G}(\phi)$. Let again $x \in G$ and $x N \in C_{G / N}(A)$. Then $\left(x^{-\phi} x\right)^{\frac{1}{2}} \in N$. As above we have

$$
x=x\left(x^{-\phi} x\right)^{-\frac{1}{2}}\left(x^{-\phi} x\right)^{\frac{1}{2}} \text { and } y=x\left(x^{-\phi} x\right)^{-\frac{1}{2}} \in H .
$$

It is easy to see that

$$
y(H \cap N) \in C_{H / H \cap N}(A) .
$$

Since the automorphism group of $H$ induced by $A$ has order strictly less than $|A|$, we are in a position to apply the induction hypothesis. This yields that $y=h t$, where $h \in C_{H}(A)$, $t \in H \cap N$. Recall that $x=y\left(x^{-\phi} x\right)^{\frac{1}{2}}$. It follows that $x N \in C_{G}(A) N / N$.

Thus, we showed that $C_{G / N}(A) \leq C_{G}(A) N / N$. The reverse inclusion is obvious.

Let now $G$ be any group and $p$ a prime. For $i \geq 1$ set

$$
D_{i}(G)=\prod_{j p^{k} \geq i} \gamma_{j}(G)^{p^{k}},
$$

where $\gamma_{j}(G)$ stands for the $j$-th term of the lower central series of $G$ and for any subgroup $H \leq G$ the symbol $H^{\text {pk }}$ denotes the subgroup of $H$ generated by the set $\left\{h^{p k} \mid h \in H\right\}$. It follows that $\gamma_{i}(G) \leq D_{i}$ for all $i \geq 1$ and that $\left(D_{i}(G)\right)_{i \geq 1}$ is a descending series of characteristic subgroups of $G$. This series is called the Lazard $p$-series of $G$.

Lemma $2.3[9$, p. 250]. For any group $G$ and all $i, j \geq 1$ we have

$$
\left[D_{i}(G), D_{j}(G)\right] \leq D_{i+j}(G) .
$$

The above lemma shows that the Lazard $p$-series $\left(D_{i}(G)\right)_{i \geq 1}$ is a strongly central series of $G$ in the sense of [9].

Given a group $G$ and a prime $p$ we shall associate the Lie algebra $L_{p}(G)$ to $G$. Consider the series

$$
G=D_{1}(G) \geq D_{2}(G) \geq \ldots
$$


For all $i \geq 1$ the factor group $D_{i}(G) / D_{i+1}(G)$ can be viewed as a vector space over the field with $p$ elements $\mathbb{F}_{p}$. Let $L(G)$ denote their direct sum,

$$
L(G)=\bigoplus_{i=1}^{\infty} D_{i}(G) / D_{i+1}(G)
$$

To avoid overloading the notation, we write $D_{i}$ for $D_{i}(G)$. For arbitrary cosets $a D_{i+1} \in D_{i} / D_{i+1}$ and $b D_{j+1} \in D_{j} / D_{j+1}$ we define a bracket product

$$
\left[a D_{i+1} ; b D_{j+1}\right]=[a, b] D_{i+j+1} .
$$

where $[a, b]$ denotes the group commutator $a^{-1} b^{-1} a b$. Lemma 2.3 implies that the product above is well defined in the sense that the right-hand side of 2.4 does not depend on the coset representatives $a, b$. Extending now the product 2.4 linearly to the whole $L(G)$, we give $L(G)$ the structure of a Lie algebra over the field $\mathbb{F}_{p}$. The subalgebra of $L(G)$ generated by $D_{1} / D_{2}$ will be denoted by $L_{p}(G)$.

If $G$ is a finitely-generated residually-finite $p$-group such that $L_{p}(G)$ satisfies a non-trivial polynomial identity, then by a deep result of Zelmanov $G$ is finite [15, Theorem 1.6].

Proposition B. Let $G$ be a periodic residually-nilpotent group admitting a fixedpoint-free automorphism $\phi$ of order $2^{n}$. Then $G$ is locally finite.

Proof. We may assume that $G$ is a finitely-generated $p$-group. Obviously $\phi$ induces an automorphism of the Lie algebra $L_{p}(G)$. By Lemma $2.1 p$ is odd, so Lemma 2.2 guarantees that $\phi$ induces a fixed-point-free automorphism of every quotient $D_{i}(G) / D_{i+1} ; i=1,2, \ldots$. This yields that the induced automorphism of $L_{p}(G)$ has no non-zero fixed element. A theorem of Kreknin [11] now tells us that $L_{p}(G)$ is solvable. Hence by Zelmanov's Theorem cited above [15] $G$ must be finite.

\section{Proof of Theorem $A$}

Let $G$ be an arbitrary periodic group. We denote by $|x|$ the order of element $x \in G$. Set $T_{1}(G)=G$, and for $i=1,2, \ldots$ define

$$
T_{i+1}=\left\langle[x, y] ; x, y \in T_{i}(G),(|x|,|y|)=1\right\rangle .
$$

Obviously we have

$$
T_{1}(G) \geq T_{2}(G) \geq \cdots \geq T_{i}(G) \geq T_{i+1}(G) \geq \ldots
$$

Lemma 3.1. Let $G$ be a periodic group, $N$ a normal subgroup of $G$. Then for any positive integer $k$ we have 


$$
T_{k}(G / N)=T_{k}(G) N / N
$$

Proof. Since $T_{k+1}(G)=T_{2}\left(T_{k}(G)\right)$, it suffices to prove the lemma only for $k=2$.

Certainly, if $x$ and $y$ have relatively prime orders in $G$, then $x N$ and $y N$ have relatively prime orders in $G / N$. Therefore the inclusion $T_{2}(G / N) \geq T_{2}(G) N / N$ is obvious.

To prove that $T_{2}(G / N) \leq T_{2}(G) N / N$ let us take $x N, y N \in G / N$ of relatively prime orders in $G / N$ and show that $[x, y] \in T_{2}(G) N$.

Let $\pi_{1}$ be the set of prime divisors of $|x N|$ and $\pi_{2}$ the set of prime divisors of $|y N|$. By assumption $\pi_{1} \cap \pi_{2}=\emptyset$. Let $|x|=m_{1}, n_{1},|y|=m_{2} n_{2}$, where $n_{1}$ is the maximal $\pi_{1}$-divisor of $|x|$ and $n_{2}$ is the maximal $\pi_{2}$-divisor of $|y|$. Then $\left\langle x^{m_{1}} N\right\rangle=\langle x N\rangle$ and $\left\langle y^{m_{2}} N\right\rangle=\langle y N\rangle$. Therefore there exist integers $i, j$ such that $x \in x^{i m_{1}} N$, and $y \in y^{j m_{2}} N$. The orders of $x^{i m_{1}}$ and $y^{j m_{2}}$ are relatively prime. Therefore

$$
[x, y] \in\left[x^{i m_{1}}, y^{j m_{2}}\right] N \in T_{2}(G) N,
$$

as required.

Given a periodic group $G$, let $t(G)$ denote the minimal number $i$ (possibly $\infty$ ) such that $T_{i+1}(G)=1$. Recall that if $G$ is a finite solvable group, then the Fitting height $h(G)$ of $G$ is defined as follows. Let $F(G)$ denote the Fitting subgroup of $G$, i.e., the subgroup generated by all normal nilpotent subgroups of $G$. Set

$$
F_{0}(G)=1, F_{i+1}(G) / F_{i}(G)=F\left(G / F_{i}(G)\right), i=1,2, \ldots
$$

Then $h(G)$ is the minimal number $h$ such that $F_{h}(G)=G$.

Lemma 3.2. Let $G$ be a finite solvable group. Then $t(G)=h(G)$.

Proof. Let $N$ be the minimal normal subgroup of $G$ such that $G / N$ is nilpotent. Then, obviously, $T_{2}(G)=N$. Now use induction on $t(G)$ along with the equalities $t\left(T_{2}(G)\right)=t(G)-1$ and $h(N)=h(G)-1$.

Lemma 3.3. Let $G$ be a periodic residually-finite group admitting a fixed-point-free automorphism $\phi$ or order $2^{n}$. Then $t(G) \leq n$.

Proof. Suppose that $t(G) \geq n+1$, i.e., $T_{n+2} \neq 1$. Since $G$ is residually-finite, there exists a $\phi$-invariant normal subgroup $N$ of finite index in $G$ such that $T_{n+2}(G) \notin N$. By Lemma $3.1 T_{n+2}(G / N) \neq 1$. We remark that by 2.1 and $2.2 G / N$ is a finite group of odd order on which $\phi$ acts without non-trivial fixed points. By a result of Th. Berger [3] $h(G / N) \leq n$. Now the previous lemma yields $t(G / N) \leq n$, a contradiction. 
Proof of Theorem A. Given an almost regular element of order $2^{n}$ in a periodic residually-finite group $G$, let $\phi$ denote the inner automorphism of $G$ induced by this element. Of course, without any loss of generality we may assume that $G$ is finitelygenerated. Since $C_{G}(\phi)$ is finite and $G$ is residually-finite, there exists a normal $\phi$-invariant subgroup $H$ of $G$ such that $|G: H|<\infty$ and $H \cap C_{G}(\phi)=1$. By Lemma 2.1 $H$ is a $2^{\prime}$-group. Applying Lemma 3.3 with $H$ in place of $G$, we conclude that $t=t(H) \leq n$. Suppose first that $t=1$. In this case $H$ is a direct product of maximal $p$-subgroups. Proposition B yields that each $\phi$-invariant $p$-subgroup of $H$ is locally finite and, therefore, so is $H$. Since $H$ is of finite index in $G$, it follows that $G$ is locally finite and we are done.

Thus, without loss of any generality we may assume that $t \geq 2$ and use induction on $t$. By the induction hypothesis assume that $T_{2}(H)$ is locally finite. Set $N=T_{2}(H)$. Then $H / N$ is a direct product of its maximal p-subgroups. By Lemma 2.2 this group admits a fixed-point-free 2-automorphism. To use Proposition $B$ now we need to know that $H / N$ is residually-finite: but this can be false. So, let $H_{0}$ be the intersection of all normal subgroups of finite index in $H$ which contain $N$. Then $H / H_{0}$ is a direct product of its maximal $p$-subgroups (because $N \leq H_{0}$ ) and is residually-finite (by the definition of $H_{0}$ ). Lemma 2.2 shows that $H / H_{0}$ admits a fixed-point-free automorphism whose order divides $2^{n}$. Now Proposition B implies that $H / H_{0}$ is locally finite. Since $H$ is a subgroup of finite index of a finitely-generated group, $\mathrm{H} / \mathrm{H}_{0}$ also is finitely-generated. Thus, we derive that $H / H_{0}$ is finite. In particular each subgroup of $H_{0}$ having finite index in $H_{0}$ has also finite index in $H$. Now the definition of $H_{0}$ implies that for any subgroup $K$ of finite index in $H_{0}$ we have $N K=H_{0}$. It follows that each finite quotient of $H_{0}$ is isomorphic to a finite quotient of $N$. Since $N=T_{2}(H)$, it follows that $t(N)=t-1$. We conclude now that for any finite quotient $\bar{H}$ of $H_{0}$ we have $t(\bar{H}) \leq t-1$. Since $H_{0}$ is residually-finite, Lemma 3.1 yields now that $t\left(H_{0}\right) \leq t-1$. By the induction hypothesis we derive now that $H_{0}$ is locally finite. Due to the fact that $H_{0}$ has finite index in $G$ it follows that $G$ is locally finite. The proof is complete.

\section{REFERENCES}

1. S. V. AleshiN, Finite automata and the Burnside problem for periodic groups, Math. Notes 11 (1972), 199-203.

2. V. V. BelyaEv, Groups with almost regular involution, Algebra and Logic 26 (1987), 315-317.

3. Th. Berger, Nilpotent fixed point free automorphism groups of solvable groups, Math. Z. 131 (1973), 305-312.

4. E. S. GoLod, On nil-algebras and residually finite groups, Izv. Akad. Nauk SSSR, Ser. Mat. 28 (1964), 273-276.

5. R. I. GrigorchuK, On the Burnside problem for periodic groups, Funct. Anal. Appl. 14 (1980), 53-54.

6. N. GUPTA and S. SIDKI, On the Burnside problem for periodic groups, Math. $Z$. 182 (1983), 385-386. 
7. B. Hartley, Centralizers in locally finite groups, in Proc. Conf. Group Theory, Brixen/ Bressanone 1986, (Lecture Notes in Math. 1281, Springer, 1987), 36-51.

8. B. HaRtLey, Simple locally finite groups, in Finite and locally finite groups (Proc. Conf. Istanbul, 1994, Kluwer Academic Publishers, 1995), 1-44.

9. B. HUPPERT and N. BlackbURN, Finite groups II (Springer, Berlin, 1982).

10. E. I. KhuKhro, Groups and Lie rings admitting almost regular automorphisms of prime order, in Proc. Int. Conf. Theory of Groups, Bressanone/Brixen, 1989 (Suppl. Rend. Circ. Mat. Palermo, 1990), 183-192.

11. V. A. KREKNIN, Solvability of Lie algebras with a regular automorphism of finite period, Soviet Mat. Dokl. 4 (1963), 683-685.

12. A. YU. OL'ShanskiI, On residualing homomorphisms and $G$-subgroups of hyperbolic groups, Internat. J. Algebra Comput. 3 (1993), 365-409.

13. V. I. Sushchansky, Periodic p-elements of permutations and the general Burnside problem, Dokl. Akad. Nauk SSSR 247 (1979), 447-461.

14. V. P. Šunkov, On periodic groups with an almost regular involution, Algebra and Logic 11 (1972), 260-272.

15. E. Zelmanov, Lie ring methods in the theory of nilpotent groups, in Groups '93, Galway-St Andrews (London Math. Soc. Lecture Note Series, 212, 1995), 567-586.

Department of Mathematics

UNIVERSITY OF BRASILIA

70.919 BRASILIA - DF

BRAZIL 